\title{
Fable: Design of a Modular Robotic Playware Platform
}

Pacheco, Moises; Moghadam, Mikael; Magnússon, Arnpór; Silverman, B.; Lund, Henrik Hautop ; Christensen, David Johan

\section{Published in:}

Proceedings of the IEEE International Conference on Robotics and Automation

Link to article, DOI:

10.1109/ICRA.2013.6630627

Publication date:

2013

Link back to DTU Orbit

Citation $(A P A)$ :

Pacheco, M., Moghadam, M., Magnússon, A., Silverman, B., Lund, H. H., \& Christensen, D. J. (2013). Fable: Design of a Modular Robotic Playware Platform. In Proceedings of the IEEE International Conference on Robotics and Automation IEEE. https://doi.org/10.1109/ICRA.2013.6630627

\section{General rights}

Copyright and moral rights for the publications made accessible in the public portal are retained by the authors and/or other copyright owners and it is a condition of accessing publications that users recognise and abide by the legal requirements associated with these rights.

- Users may download and print one copy of any publication from the public portal for the purpose of private study or research.

- You may not further distribute the material or use it for any profit-making activity or commercial gain

- You may freely distribute the URL identifying the publication in the public portal 


\title{
Fable: Design of a Modular Robotic Playware Platform
}

\author{
M. Pacheco*, M. Moghadam*, A. Magnússon*, B. Silverman ${ }^{\dagger}$, H. H. Lund* and D. J. Christensen*
}

\begin{abstract}
We are developing the Fable modular robotic system as a playware platform that will enable non-expert users to develop robots ranging from advanced robotic toys to robotic solutions to problems encountered in their daily lives. This paper presents the mechanical design of Fable: a chain-based system composed of reconfigurable heterogeneous modules with a reliable and scalable connector. Furthermore, this paper describes tests where the connector design is tested with children, and presents examples of a moving snake and a quadruped robot, as well as an interactive upper humanoid torso.
\end{abstract}

\section{INTRODUCTION}

Construction toys (e.g. LEGO) have the potential to facilitate creativity and learning by capturing the user in a mental state of focused play. In this work we explore modular playware as mechatronic construction toys that are animated and which become playfully alive as they are assembled from individual modules. To achieve this, we are developing novel interactive technology utilizing experiences from modular robotics, embodied artificial intelligence, and human-robot interactions, in order to create technological products that will motivate users to interact and play.

In this paper we describe the mechanical design of the Fable modular robotic system. The modules comprising this system can be combined by a user to create various robotic creatures, such as robotic snakes, walking robots, vehicles, humanoids, or other fantasy creatures. The longterm vision is to transform the development of robots from something done solely by experts to something so widely available, easily accessible, and motivating, that anybody is able to realize their ideas for robotic artifacts or solutions to problems encountered in their own lives.

Modular robotic systems are collections of simple robotic modules that can attach and detach from each other to form virtually endless different configurations [24], [25]. Several systems are designed to support self-reconfiguration, e.g. [6], [13], while other systems are designed for rapid robot prototyping [26], constructing walking robots [7], adaptive furniture [22], or space exploration [18].

This paper explores modular robotic playware, which are modular robotic systems designed to enable a user to construct artifacts for playful activities [8]. The modular robotic playware approach suggests that, the best way to allow users

*Technical University of Denmark, Center for Playware, DTU Electrical Engineering, Elektrovej, building 325, DK-2800 Kgs. Lyngby, Denmark $\{$ mpac $|\mathrm{mikm}| \mathrm{hhl} \mid \mathrm{dj} \mathrm{chr}\}$ delektro.dtu.dk, arnthormegmail.com

$\dagger$ Playful Invention Company, Montreal, Quebec, Canada bss@media.mit.edu to develop robotic systems is through contextualized handson problem solving, which permits the users to work directly with technological building blocks in their own context. By the free manipulation of combining the technological building blocks, the user is developing technological prototypes $\mathrm{him} /$ herself, and these technological prototypes can be tested immediately as they are being constructed by the user in the user's context [9]. In general, the modular system becomes an object to think with, and the modularity invites the user to perform physical manipulation and reconfiguration [10].

Examples of modular robotic playware include the Topobo system which enables the user to record and playback motor sequences by programming-by-demonstration [16] and the roBlocks (now Cubelets) which utilize a programmingby-building strategy where the behavior is emergent from modules interactions with each other and their environment [20]. Further, the LEGO Mindstorms is a robotic construction kit that enables direct-user-programming of LEGO models equipped with actuation and sensing. Similar to these examples the Fable system aims to enable everyone to become a robot designer and become motivated to be creative, explore, construct, reflect, iterate, play and share.

We anticipate that the Fable system, when fully developed, will complement the previous playware examples in several novel ways: i) New robots with powerful actuation and sensing can be constructed in seconds, ii) robots can be programmed in several ways depending on the preference of the user (e.g. programming-by-building, programmingby-demonstration, programming-with-tags, and direct-userprogramming), iii) socially interactive and adaptive robots can be de developed based on smart sensor modules that provide higher-level information about the user. To facilitate human-robot interaction we are inspired by robots such as Probo [17], Huggable [23], and Kismet [2].

In the rest of this paper we describe the mechanical design (Sec. II) and summarize the electronic design (Sec. III) of the Fable system. The software architecture is described in Sec. IV. Afterwards Sec. V describes a user test with the connector design as well as tests with moving and interactive robots.

\section{MECHANICAL DESIGN}

The Fable system is designed as a modular robotic playware platform, suitable for creating interactive constructions such as robotic toys. A Fable robot consists of heterogeneous modules which provide the necessary functionality to be able to perform various tasks such as sensing the user, its environment, actuating joints for movement and producing sounds. In the design of the modules we have tried to 


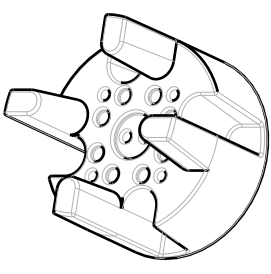

(a) Small connector

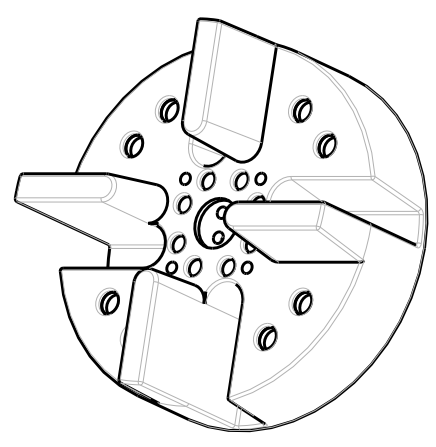

(b) Medium connector

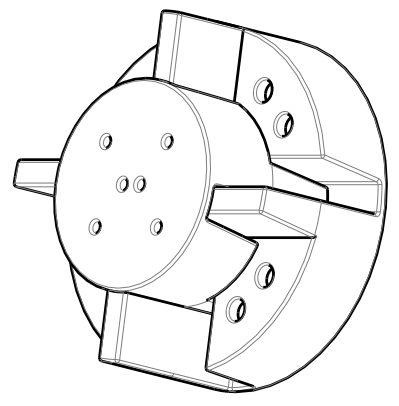

(c) Compatibility of two different sized connectors

Fig. 1. Connector design: With the current design any size is possible and compatible with the rest, having only as a lower limit the small size connector diameter.

make them appropriate for interaction with non-technical users and children. Mechanical magnetic connectors allow a solid attachment and detachment between modules for rapid construction of robotic morphologies. The morphologies can be rearranged in numerous configurations based on joints, branching, and termination modules. The modules are designed to be aesthetically pleasing by utilizing rounded and organic shapes. Further, the connectors are scalable so as to allow modules of different sizes to be combined. The system is chain-based since we observe that this often simplifies the assembly of functional robots (compared to lattice based systems). The mechanical design of the Fable system is inspired by several existing modular robotic systems, such as the Cubelets [12], Topobo [16], ATRON [14], CKBot [19], and Roombots [21] and share some characteristics with these systems. This section describes the mechanical design of connectors and some different categories of modules (i.e. structuring, joint, branching, and termination).

\section{A. Connectors}

In a modular system, connectors are the only interface between neighbouring robots. They are responsible for maintaining a given configuration, as well as for allowing it to change. The following list sums up the requirements for the connector design.

- Strong connection and yet easy enough for children to disconnect.

- Robust to wear and tear.

- Transfer communication signals.

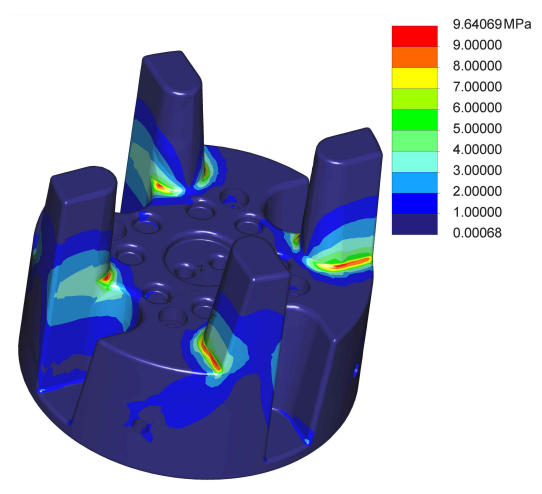

(a) Small connector

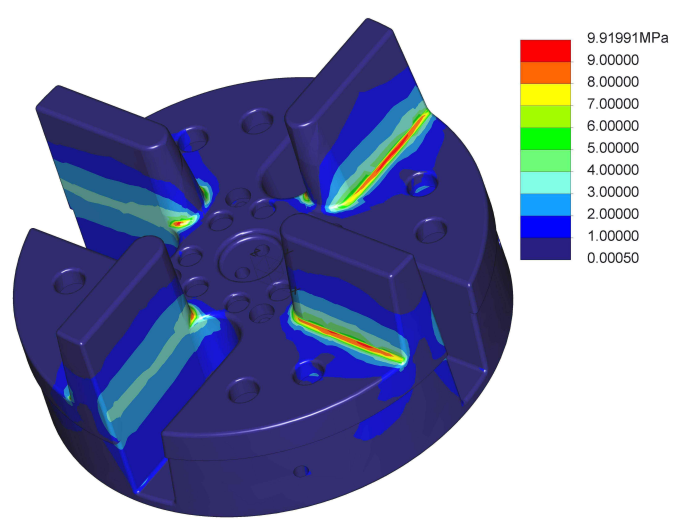

(b) Medium connector

Fig. 2. Von Misses Stress analysis on a PLA design with an applied pressure of $0.45 \mathrm{MPa}$ per flange on the medium size connector and 0.55 MPa per flange on the small size connector.

- Genderless.

- Multiple connection configurations.

- Scalable to maintain compatibility with larger and smaller modules.

Figures 1(a) and 1(b) show the overall design of two different connector sizes: small and medium. From these images it can be seen that there is a repetetive pattern every $90^{\circ}$, this pattern gives the user four possibilities of establishing a connection. Each of the patterns is conformed by an arch of magnets, a flange and a hole. Where each arch is conformed by a set of two magnets with alternating $\mathrm{S}-\mathrm{N}$ poles. An extra ring of magnets was placed on the medium sized connector to ensure compatibility between both sizes, as seen on Figure 1(c). The flanges mechanically lock the connection against twisting and bending and only allow detachment by pulling on the axis perpendicular to the connecting surfaces. In this manner the magnetic force is concentrated on the same axis as the disconnection axis, this enables a strong connection between modules, requiring a force of $12 \mathrm{~N}$ or $30 \mathrm{~N}$ to disconnect the small or medium size respectively. As a result of implementing a simple design we allow users, even as young as 6 years old, to establish a connection ( further details are given in Sec. V). The European Standard for Safety of Toys requires a toy to withstand a torque of $0.34 \mathrm{Nm}$ for a ten second interval in a clockwise manner as well as in the opposite direction 
[1]. In order to verify this, a stress analysis was made by applying a pressure of $0.55 \mathrm{MPa}$ per flange on a $56 \mathrm{~mm}^{2}$ area on the small size connector and a pressure of $0.45 \mathrm{MPa}$ per flange on a $196 \mathrm{~mm}^{2}$ area on the medium size connector, the results obtained are displayed on Figure 2. This implies that it is necessary to apply a torque of $2.094 \mathrm{Nm}$ or $8.11 \mathrm{Nm}$ in order to break the mechanical lock of the small or medium size connector respectively. Furthermore, as a tensile test the European Standard requires a dead weight of at least $90 \mathrm{~N}$ when the largest accessible dimension is greater than $6 \mathrm{~mm}$. Both requirements are fulfilled by the design.

\section{B. Modules}

Joint modules (JM) are robotic modules used to enable locomotion as well as interaction with the robots environment. Each articulation of the creature may have various requirements, therefore different joints have to be created. There are currently 3 different configurations of Joint modules: 1 DoF, 2 DoF and 3 DoF. The goal with all JM is to have very similar dimensions, if not identical. Figures 3(d)3(f) show the designs of the 3 types of JM. All JMs have a maximum diameter of $87 \mathrm{~mm}$.

Branching modules (BM) are units used to connect several modules together in tree-like configurations. We currently have three designs of BM. The first one being the 2 Way Branch, shown on Figure 3(a). This module enables communication between two modules. The second one being the 3 Way Branch, shown on Figure 3(b), enables communication between 3 modules by establishing connections at $120^{\circ}$ intervals. The third one being the 4 Way Branch, as seen on Figure 3(c), which enables 4 modules to communicate with each other.

Termination Modules (TM) are designed to close off open connectors on a robot. TM's may add a visual expression, additional sensors, or actuators (e.g. grippers or wheels). We are currently developing several TM's, including a foot module, to enable walking creatures to walk more efficiently; a wheel module and a vision module, which makes use of Asus' Xtion PRO LIVE to equip the system with stereo vision.

Table I summarizes the characteristics of the modules shown in Figure 3.

\begin{tabular}{|c|c|c|c|}
\hline Module & Height $(\mathrm{mm})$ & Weight $(\mathrm{g})$ & DoF \\
\hline 2 Way Branch & 142 & 300 & 0 \\
\hline 3 Way Branch & 130 & 350 & 0 \\
\hline 4 Way Branch & 142 & 450 & 0 \\
\hline 1 DoF JM & 210 & 450 & 1 \\
\hline 2 DoF JM & 244 & 500 & 2 \\
\hline 3 DoF JM & 275 & 550 & 3 \\
\hline
\end{tabular}

TABLE I

Module Characteristics, ALL WEIGHTS GIVEN ARE INCLUding BATTERY AND ELECTRONICS

\section{ELECTRONIC DESIGN}

The module's electronic board has an Atmel ATMega2561 microcontroller running at $8 \mathrm{MHz}$ with $256 \mathrm{~Kb}$ of FLASH

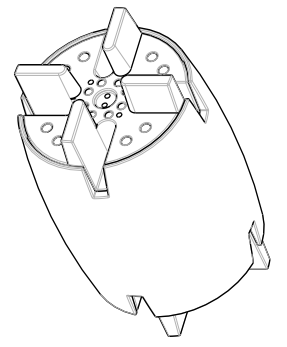

(a) 2 Way Branch

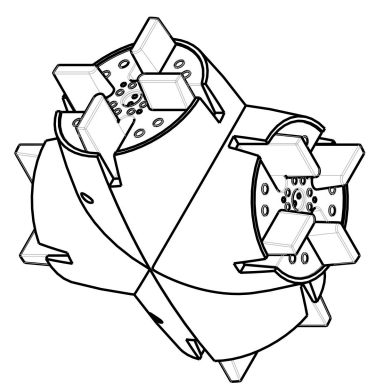

(c) 4 Way Branch

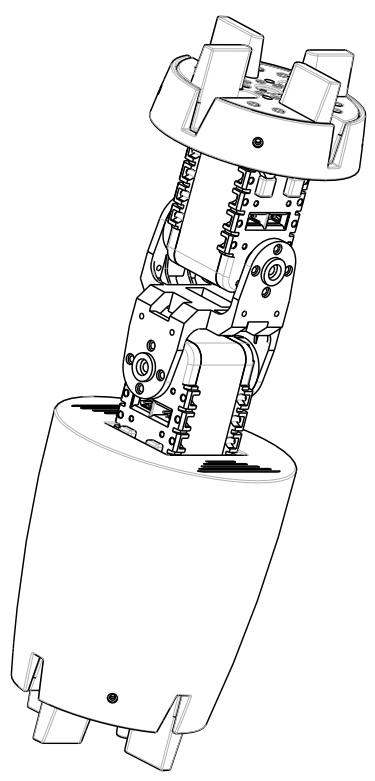

(e) 2 DoF JM

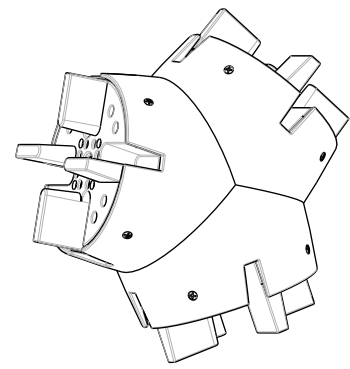

(b) 3 Way Branch

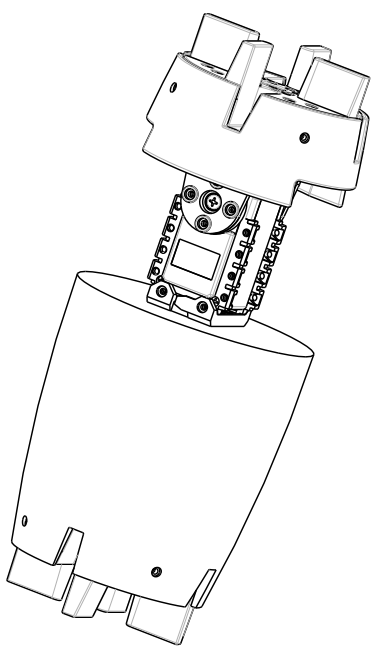

(d) 1 DoF JM

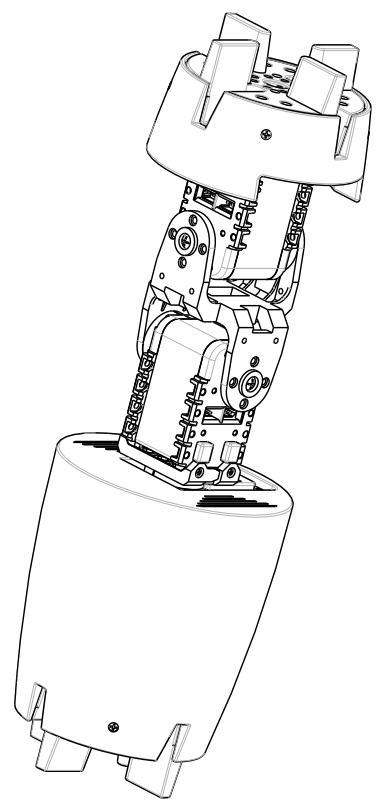

(f) $3 \mathrm{DoF} J \mathrm{M}$
Fig. 3. Fable Modules 
and $8 \mathrm{~Kb}$ of RAM. The electronic boards have a connector to power and can control several daisy chained AX-12A Dynamixel servos. Further, the boards have four IR channels (half-duplex) used for neighbour-to-neighbour communication. In addition, each board can be equipped with an XBee dongle for wireless communication between modules or to a PC. All of the electronic boards have an accelerometer, a gyroscope, and the possibility to attach additional sensors and a small speaker for simple sound output. The boards are powered from a three cell $11.1 \mathrm{~V}$ lithium-polymer rechargeable battery with a capacity of $1000 \mathrm{mAh}$. The microcontroller can be programmed externally without disassembling the modules. These custom made electronic boards are in the process of being integrated into the system. For the experiments presented in Section $\mathrm{V}$, we used a compatible embedded system (CM-510 controller box) for centralized control of the modules. However, the software and control architecture used is fully functional and will be ported directly to electronic boards for distributed control.

\section{SOFTWARE ARCHITECTURE}

Ideally application programming for the Fable system should be open and accessible to non-expert users (e.g. designers, students and even kids). Thus the programming tools must abstract away many of the low level details of sensors, motors and distributed processing. Therefore the Fable software architecture has both a low-level firmware part as well as a virtual machine to provide high-level programming of robot applications.

The embedded microcontrollers in the Fable modules are running a low-level software system which has been developed primarily in $\mathrm{C}$. In order to ease the development of the embedded firmware we utilize the Assemble-andAnimate framework (ASE) [3]. This gives us a high level of abstraction, a large library of components and algorithms and an asynchronous event based framework.

For inexperienced programmers the $\mathrm{C}$ programming language can be too complex to utilize. We have therefore chosen to provide the LOGO programming language for application development. LOGO is an educational programming language which provide a higher level of abstraction and a more natural English-like syntax than C (e.g. no braces). The Fable firmware includes a virtual machine (VM) that runs as a (non-blocking) process in the ASE scheduler and can execute LOGO applications compiled to byte-code. The VM allows the user to upload LOGO byte-code to the modules through a serial connection or a USB flash drive. Running in a VM also means that the LOGO code can run on the modules more safely since the VM limits the program's access to the systems resources. Domain specific primitives with a higher abstraction level are included as part of the provided LOGO language. These features enable the user to more quickly develop an application and upload it to the robot.

We use a restricted version of LOGO for embedded devices, called PicoLOGO developed by Brian Silverman [5]. PicoLOGO has a reduced set of available instructions and is currently limited to only integer type variables. There is a performance penalty when executing LOGO code in the virtual machine, compared to the equivalent $\mathrm{C}$. Microbenchmarks indicate a penalty factor of approximately 9 .

For more advanced processing, than possible or feasible on an 8-bit mirocontroller, we utilize the Robot Operating System (ROS) [15]. ROS gives us access to a large set of software libraries such as OpenCV. The software architecture is such that we run ROS on a server which provides services (over a wired or wireless serial connection) to the LOGO code running on the modules. This architecture has for instance enabled us to develop a humanoid model, from the modules, that can speak, detect faces, and mimic postures from humans using a motion sensing input device [11].

\section{TESTS}

This section describes several tests performed with the Fable system: 1) a user test performed with children to validate the usability of the connector design, 2) a snake and 3) a quadruped robot to validate the systems ability to construct mobile robots and 4) a simple humanoid upper torso to illustrate the construction of interactive robotic artefacts.

Connectors. It was stated as a design requirement, in Section II, that children should be able to attach and detach the Fable modules. To investigate this requirement a user test was performed at an after school club in the Copenhagen area. At the time of testing, no modules had been prototyped. Instead the modules were represented by a solid PVC cylinders with a connector attached to one end. Both the medium and small sized connectors, prototyped with SLS technology, were tested. The diameters of the cylinders were $40 \mathrm{~mm}$ for the small connector and $60 \mathrm{~mm}$ for the medium, both of them having a height of $100 \mathrm{~mm}$. The connectors had magnets attached to them to help fasten the connection just as stated within the design description. The age group that was targeted for the design was ages 6 and up. The group tested consisted of 16 children. Their ages ranged between 6-10 years, where 9 out of the group were 6,1 was 7, 3 were 8,2 were 9 and 1 was 10 years old.

The children were asked to connect and disconnect the connectors several times and we observed if they encountered any difficulty. Most of the children did not have issues connecting the modules. Four kids, mainly 6 years old, had issues connecting during the first couple of tries until they figured out how to connect them. Sometimes it was sufficient for them to mimic the process, just by watching an older child succeed. We also observed that the force required for disassembly exceeded that of most children. Further tests with different connector prototypes are needed to determine an appropriate design that enables children to comfortably disconnect two modules.

Quadruped locomotion. The 8 DoF quadruped is built by connecting a 2 DoF Joint module to each end of a 4 Way Branch. The configuration weighs a total of $2024 \mathrm{~g}$, including battery and controller. 


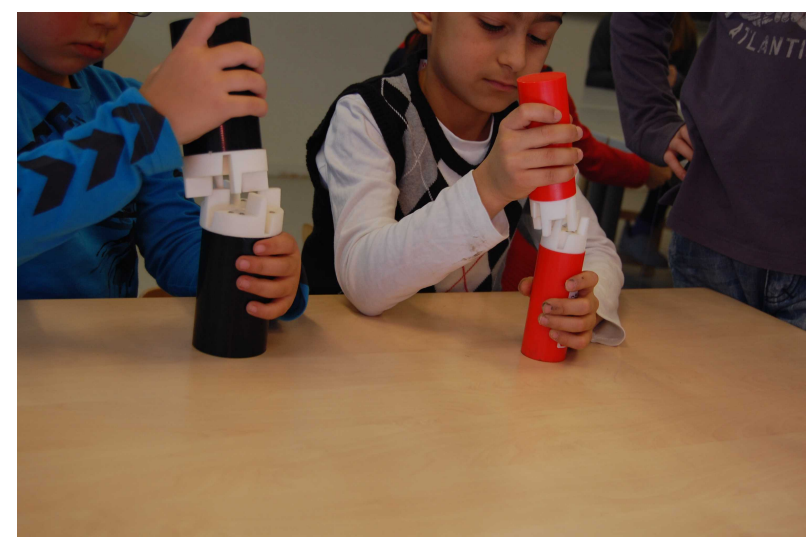

Fig. 4. Two boys connecting a medium sized connector and a small sized connector

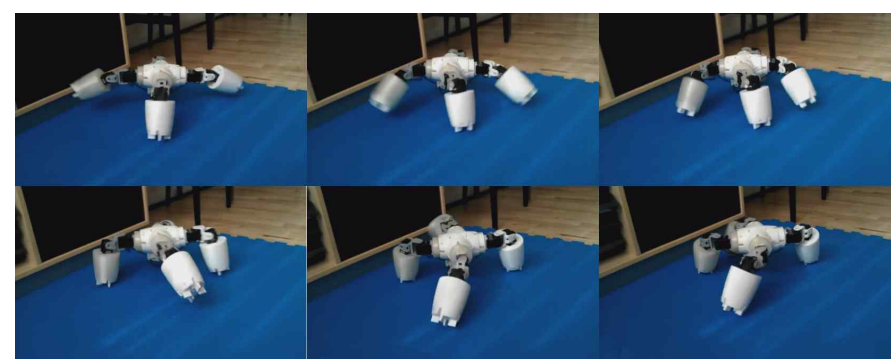

Fig. 5. Walking cycle for the quadruped configuration

A Central Pattern Generator (CPG) based controller was developed for the quadruped (based on the CPG architecture of coupled oscillators presented in previous work [3], [4]). Four gaits were implemented in the design: forward, backward, clockwise turn and counter-clockwise turn. The current gait is controlled by the analog stick of a gamepad. The gamepad is connected to a PC which has a wireless ZigBee communication link with the robot. Only a high level control signal is sent to the robot. All CPG computation was performed locally by the embedded microcontroller.

To study the robot's mobility the test setup was based on two types of floors with different friction coefficients. The first one being a foam based mat and the second one being a linoleum based floor. The walking speed was measured by attaching a string to the robots body and measuring the time it took it to pull the string a distance of $1.5 \mathrm{~m}$. The results obtained are shown on Table II. The turn speed was made by averaging the time it took the robot to make 10 full revolutions. On foam, the average turn rate was $0.18 \mathrm{rev} / \mathrm{s}$ while the average value obtained on the Linoleum was 0.15 rev/s.

We observed that the quadruped moved with a reasonable speed and turning rate compared to its size and that the CPG architecture produced natural smooth transitions when switching between gaits. Further, we encountered no issues with servos being too weak or connectors disconnecting unintentionally. Pictures from a test run is shown in Fig.

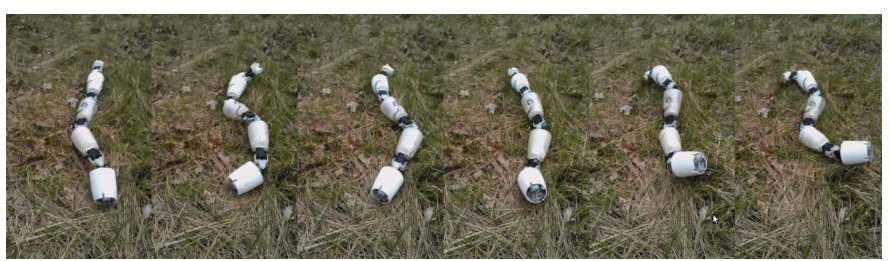

Fig. 6. Sidewinding cycle for the snake configuration

\section{$5^{1}$.}

Snake locomotion. This section describes the tests done with the snake configuration. Four 2 DoF JMs are arranged in series in order to achieve the morphology presented. This snake configuration weighs a total of $1771 \mathrm{~g}$, including the battery and electronics.

For this configuration a side-winding gait was implemented based on the same CPG architecture as for the quadruped. The frequency of the gait was controlled using the analog stick of a gamepad.

The same types of floors were used for tests as in the quadruped test, i.e. the first one being a foam based mat and the second one being a linoleum based hallway. A side winding distance of $60 \mathrm{~cm}$ was used in order to determine the speed in each scenario. It was difficult to measure more due to the fact that the mat used was relatively small and the snakes movement was not very straight and it tended to fall of the mat at longer distances. The distance was measured with two strips that marked the $60 \mathrm{~cm}$. The results obtained are shown on Table II. Ten attempts were registered in total for each scenario. The average speed for the Foam mat scenario is of $0.17 \mathrm{~m} / \mathrm{s}$ and the average speed obtained for the Linoleum is of $0.19 \mathrm{~m} / \mathrm{s}$. The results obtained in each scenario are shown on Table II.

We observed that although the snake velocity is slightly higher than the quadruped velocity the snake is much less controllable (not moving straight, flipping over). Moreover, we found no efficient gaits for turning or forward locomotion. To address these issues we will consider including the possibility of adding passive wheels to the Joint modules so that more effective gaits can be implemented.

\begin{tabular}{|l|c|c|c|c|}
\hline & \multicolumn{2}{|c|}{ Quadruped } & \multicolumn{2}{c|}{ Snake } \\
\hline Scenario/Gaits & Forward & Turn & Forward & Turn \\
\hline Foam & $0.15 \mathrm{~m} / \mathrm{s}$ & $0.18 \mathrm{rev} / \mathrm{s}$ & $0.17 \mathrm{~m} / \mathrm{s}$ & - \\
\hline Linoleum & $0.14 \mathrm{~m} / \mathrm{s}$ & $0.15 \mathrm{rev} / \mathrm{s}$ & $0.19 \mathrm{~m} / \mathrm{s}$ & - \\
\hline
\end{tabular}

TABLE II

LOCOMOTION MEASUREMENTS

Humanoid torso. To study the possibility of utilizing the Fable system in interactive toy applications a simple humanoid torso was constructed. The modules used in this test were two 2 DoF Joint Modules and one 4 Way Branch. In addition an IR distance sensor is placed to sense objects in front of the robot, see Fig. 7(a). A microphone would detect

\footnotetext{
${ }^{1}$ Videos of the different robots can be found on: http://www.youtube.com/user/centerforplayware
} 


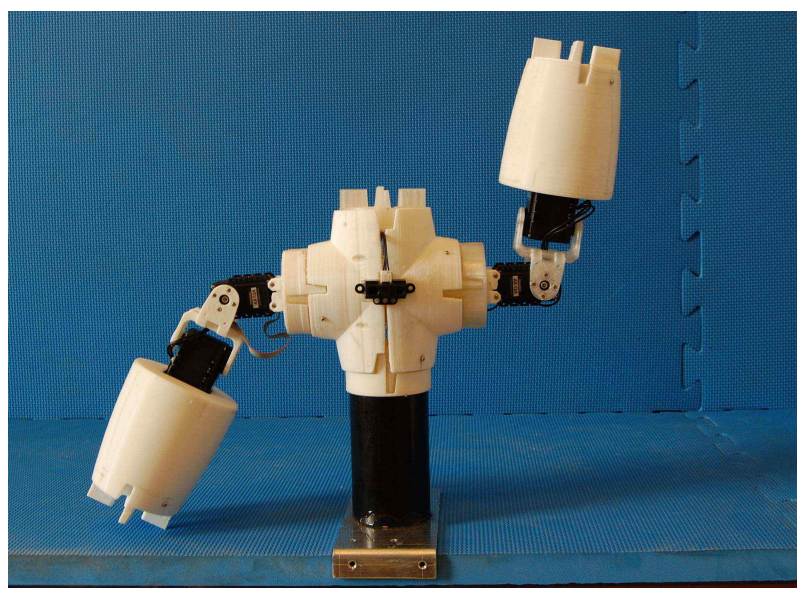

(a)

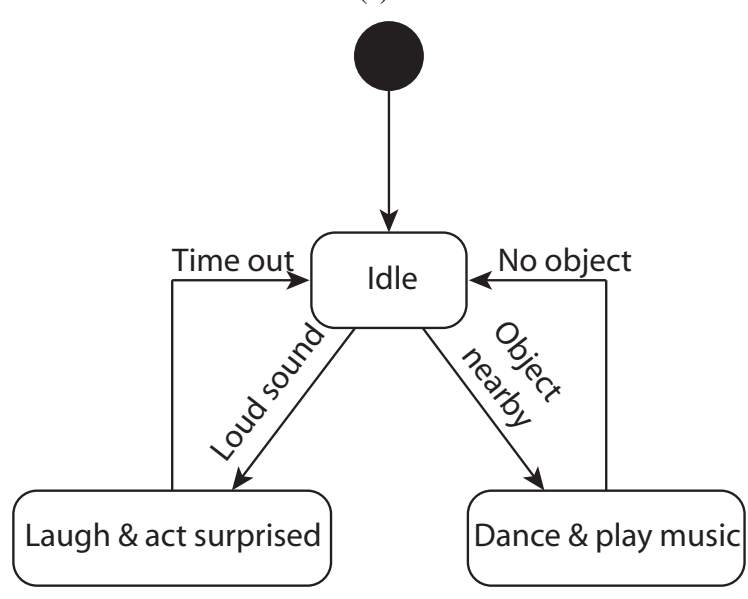

(b)

Fig. 7. (a) Humanoid Torso and (b) statechart of its controller

loud noises such as claps. Sound playback was performed by a PC controlled wireless from the robot.

A simple controller was developed for this robot, see the statechart in Fig. 7(b). A loud noise would cause the robot to make a fast jerky movement and laugh with a baby voice to act surprised. Further, if an object was detected by the distance sensor the robot would start a dance/wave and play a children's song.

We observed that the simplicity of the robot makes it feasible for such applications to be constructed and programmed by non-expert users given the necessary programming tools. However, several additional module types at different scales are required to fully be able to construct humanoid shapes with the Fable system.

Humanoid torso with gesture detection. Several playful applications have been developed to test the integration of the software architecture. For a humanoid torso a motion sensing camera module was mounted as a head of the robot. Applications were written in LOGO, compiled to byte-code, and ran in the LOGO virtual machine controlling the robot. The applications would use ROS' services, running on a server, to speak announcements and detect gestures made by the user. Two applications were made, one which would

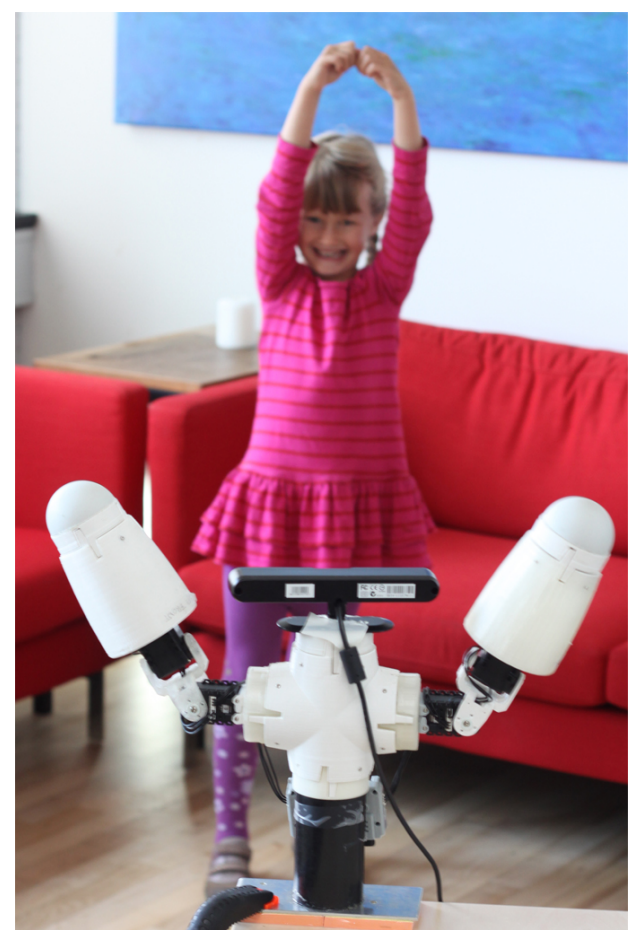

Fig. 8. 6-year old girl playing with a Fable humanoid.

mirror the users movements and one where the user should mimic the robots gestures.

The applications were tested with high school students (age 16-17) in an educational context, where the students were given exercises in programming the robot. Further, a pilot test with a young child playing with the robots has also been performed (see Fig. V). We observed that the system could be programmed by the high-school students with little training and that the child enjoyed herself while playing. We anticipate that further extensions and refinements of the modules and the software architecture will enable us and non-expert users to develop such custom playful applications.

\section{CONCLUSIONS}

In this paper we described the mechanical design of Fable which is a chain-based and heterogeneous modular robot with a reliable and scalable connector design. The purpose of Fable as a modular robotic playware platform is to enable non-technical users to create interactive robotic constructions. We described several types of modules: joint, branching, and termination. Further, we presented usability tests of the connector design which indicated that they can be handled even by young children (ages 6 and up). The mobility of the system was demonstrated by implementing a quadruped and a snake robot controlled by a central pattern generator. Finally, the potential of realizing interactive robotic constructions was demonstrated with a simple humanoid upper torso with gesture detection.

Currently we are refining and extending the Fable modules and its software architecture based on observations from robot demonstrations and user testing. We are working on 
several methods to engage the user in the development and interaction with the system, e.g. by exploring different programming paradigms (e.g. tangible programming), by taking advantage of the scalable connector design, and by enabling the system to be combined with non-module elements (such a Play-Doh or LEGO bricks).

\section{ACKNOWLEDGEMENTS}

This work was performed as part of the "Modular Playware Technology" project funded by the Danish National Advanced Technology Foundation.

\section{REFERENCES}

[1] EN 71-1:2005+A8:2009. Safety of toys - Part 1: Mechanical and physical properties. CEN, Brussels, Belgium.

[2] C. Breazeal and B. Scassellati. A context-dependent attention system for a social robot. $r n, 255: 3,1999$.

[3] D. J. Christensen, U. P. Schultz, and M. Moghadam. The assemble and animate control framework for modular reconfigurable robots. In Proceedings of the IROS Workshop on Reconfigurable Modular Robotics: Challenges of Mechatronic and Bio-Chemo-Hybrid Systems, 2011.

[4] D. J. Christensen, A. Sproewitz, and A. J. Ijspeert. Distributed online learning of central pattern generators in modular robots. In Proceedings of the 11th International Conference on Simulation of Adaptive Behavior (SAB2010), pages 402-412, Paris, France, August 2010.

[5] Playful invention company. http://www.picocricket.com/picopeople.html. Developers of PicoLOGO.

[6] H. Kurokawa, K. Tomita, A. Kamimura, S. Kokaji, T. Hasuo, and S. Murata. Distributed self-reconfiguration of M-TRAN III modular robotic system. International Journal of Robotics Research, 27(34):373-386, 2008.

[7] J. C. Larsen, R. F. M. Garcia, and K. Stoy. Increased versatility of modular robots through layered heterogeneity. In Proceedings of the ICRA Workshop on Modular Robots, State of the Art, pages 24-29, Anchorage, Alaska, May 2010.

[8] H. H. Lund. Modular interactive tiles for rehabilitation: evidence and effect. In Proceedings of the 10th WSEAS international conference on Applied computer science, ACS'10, pages 520-525, Stevens Point, Wisconsin, USA, 2010. World Scientific and Engineering Academy and Society (WSEAS).

[9] H.H. Lund. Anybody, anywhere, anytime - robotics with a social impact through a building block approach. In Proceedings of IEEE Workshop of Advanced Robotics and its Social Impact (ARSO), CA, United States, 2011.

[10] H.H. Lund and P. Marti. Designing modular robotic playware. In Robot and Human Interactive Communication, 2009. RO-MAN 2009. The 18th IEEE International Symposium on, pages 115-121. IEEE, 2009.

[11] A. Magnusson, M. Pacheco, M. Moghadam, H. H. Lund, and D.J. Christensen. Fable: Socially interactive modular robot. In Proceedings of International Symposium on Artificial Life and Robotic(AROB), 2013.

[12] Modular Robotics LLC, December 2011.

[13] E. H. Østergaard, K. Kassow, R. Beck, and H. H. Lund. Design of the ATRON lattice-based self-reconfigurable robot. Autonomous Robots, 21:165-183, 2006

[14] E. H. Østergaard, K. Kassow, R. Beck, and H.H. Lund. Design of the atron lattice-based self-reconfigurable robot. Autonomous Robots, 21(2):165-183, 2006.

[15] M. Quigley, B. Gerkey, K. Conley, J. Faust, T. Foote, J. Leibs, E. Berger, R. Wheeler, and A. Ng. Ros: an open-source robot operating system. In ICRA Workshop on Open Source Software, 2009.

[16] H. S. Raffle, A. Parkes, and H. Ishii. Topobo: a constructive assembly system with kinetic memory. In Proceedings of the SIGCHI conference on Human factors in computing systems, CHI '04, pages 647-654, New York, NY, USA, 2004. ACM.

[17] J. Saldien, K. Goris, S. Yilmazyildiz, W. Verhelst, and D. Lefeber. On the design of the huggable robot probo. Journal of Physical Agents, 2(2):3-12, 2008.
[18] B. Salemi, M. Moll, and W.M. Shen. Superbot: A deployable, multifunctional, and modular self-reconfigurable robotic system. In Intelligent Robots and Systems, 2006 IEEE/RSJ International Conference on, pages 3636-3641. IEEE, 2006.

[19] J. Sastra, S. Chitta, and M. Yim. Dynamic rolling for a modular loop robot. In Proc. of Intl. Symp. on Experimental Robotics, Rio de Janeiro Brazil, 2006.

[20] E. Schweikardt and M. D. Gross. roblocks: a robotic construction kit for mathematics and science education. In Proceedings of the 8th international conference on Multimodal interfaces, ICMI '06, pages 72-75, New York, NY, USA, 2006. ACM.

[21] A. Sproewitz, A. Billard, P. Dillenbourg, and A.J. Ijspeert. Roombotsmechanical design of self-reconfiguring modular robots for adaptive furniture. In Robotics and Automation, 2009. ICRA'09. IEEE International Conference on, pages 4259-4264. IEEE, 2009.

[22] A. Sproewitz, S. Pouya, S. Bonardi, J. van den Kieboom, R. Mockel, A. Billard, P. Dillenbourg, and A. Ijspeert. Roombots: Reconfigurable Robots for Adaptive Furniture. IEEE Computational Intelligence Magazine, special issue on Evolutionary and developmental approaches to robotics, 5(3):20-32, 2010.

[23] W. D. Stiehl, J. K. Lee, C. Breazeal, M. Nalin, A. Morandi, and A. Sanna. The huggable: a platform for research in robotic companions for pediatric care. In Proceedings of the 8th International Conference on Interaction Design and Children, IDC '09, pages 317-320, New York, NY, USA, 2009. ACM.

[24] K. Stoy, D. Brandt, and D. J. Christensen. Self-Reconfigurable Robots: An Introduction. Intelligent Robotics and Autonomous Agents series. The MIT Press, 2010.

[25] M. Yim, W-M Shen, B. Salemi, D. Rus, M. Moll, H Lipson, and E. Klavins. Modular self-reconfigurable robot systems: Challenges and opportunities for the future. IEEE Robotics \& Automation Magazine, 14(1):43-52, March 2007.

[26] M. Yim, B. Shirmohammadi, and D. Benelli. Amphibious modular robot astrobiologist. In Proceedings of SPIE Unmanned Systems Technology IX, volume 656, April 2007. 